\title{
Parallel Pointer Analysis with CFL-Reachability
}

\author{
Yu Su Ding Ye Jingling Xue \\ School of Computer Science and Engineering \\ UNSW Australia \\ \{ysu, dye, jingling $\} @$ cse.unsw.edu.au
}

\begin{abstract}
This paper presents the first parallel implementation of pointer analysis with Context-Free Language (CFL) reachability, an important foundation for supporting demand queries in compiler optimisation and software engineering. Formulated as a graph traversal problem (often with context- and field-sensitivity for desired precision) and driven by queries (issued often in batch mode), this analysis is non-trivial to parallelise.

We introduce a parallel solution to the CFL-reachability-based pointer analysis, with context- and field-sensitivity. We exploit its inherent parallelism by avoiding redundant graph traversals with two novel techniques, data sharing and query scheduling. With data sharing, paths discovered in answering a query are recorded as shortcuts so that subsequent queries will take the shortcuts instead of re-traversing its associated paths. With query scheduling, queries are prioritised according to their statically estimated dependences so that more redundant traversals can be further avoided. Evaluated using a set of 20 Java programs, our parallel implementation of CFL-reachability-based pointer analysis achieves an average speedup of $16.2 \mathrm{X}$ over a state-ofthe-art sequential implementation on $16 \mathrm{CPU}$ cores.
\end{abstract}

\section{INTRODUCTION}

Pointer (or alias) analysis, which is an important component of an optimising or parallelising compiler, determines statically what a pointer may point to at runtime. It also plays an important role in debugging, verification and security analysis. Much progress has been made to improve the precision and efficiency of pointer analysis, across several dimensions, including field-sensitivity (to match field accesses), contextsensitivity (to distinguish calling contexts) and flow-sensitivity (to consider control-flow). In the case of object-oriented programs, e.g., Java programs, both context- and field-sensitivity are considered to be essential in achieving a good balance between analysis precision and efficiency [5].

Traditionally, Andersen's algorithm [2] has been frequently used to discover the points-to information in a program (e.g., in $\mathrm{C}$ and Java). Andersen's analysis is a whole-program analysis that computes the points-to information for all variables in the program. This algorithm has two steps: finding the constraints that represent the pointer-manipulating statements and propagating these constraints until a fixed point is reached.

For many clients, such as debugging [17], [18], [19] and alias disambiguation [21], however, the points-to information is needed on-demand only for some but not all variables in the program. As a result, there has been a recent focus on demanddriven pointer analysis, founded on Context-Free Language (CFL) reachability [15], which only performs the necessary work to compute the points-to information requested in a graph representation of the program [6], [16], [17], [18], [19], [26],
[27]. By computing the points-to information of some (instead of all variables as in whole-program Andersen's analysis), demand-driven analysis can be performed both context- and field-sensitively to achieve better precision more scalably than Andersen's analysis, especially for Java programs.

However, precise CFL-reachability-based analysis can still be costly when applied to large programs. In the sequential setting, there are efforts for improving its efficiency for Java programs by resorting to refinement [18], [19], summarisation [17], [26], incrementalisation [6], [16], pre-analysis [25] and ad-hoc caching [18], [25]. However, multicore platforms, which are ubiquitous nowadays, have never been exploited for accelerating the CFL-reachability-based analysis.

There have been a few parallel implementations of Andersen's pointer analysis on multi-core CPUs [3], [8], [9], [14], GPUs [7], [10], and heterogeneous CPU-GPU systems [20], as compared in detail in Table II. As Andersen's analysis is a whole-program pointer analysis, these earlier solutions cannot be applied to parallelise demand-driven pointer analysis.

Despite the presence of redundant graph traversals both within and across the queries, parallelising CFL-reachabilitybased pointer analysis poses two challenges. First, parallelisminhibiting dependences are introduced into its various analysis stages since processing a query involves matching calling contexts and handling heap accesses via aliased base variables (e.g., $p$ and $q$ in $x=p . f$ and $q . f=y$ ). Second, the new pointsto/alias information discovered in answering some queries during graph traversals is not directly available to other queries on the (read-only) graph representation of the program.

In this paper, we describe the first parallel implementation of this important analysis for Java programs (although the proposed techniques apply equally well to C programs [27]). We expose its inherent parallelism by reducing redundant graph traversals using two novel techniques, data sharing and query scheduling. With data sharing, we recast the original graph traversal problem into a graph rewriting problem. By adding new edges to the graph representation of the program to shortcut the paths traversed in a query, we can avoid retraversing (redundantly) the same paths by taking their shortcuts instead when handling subsequent queries. With query scheduling, we prioritise the queries to be issued (in batch mode) according to their statically estimated dependences so that more redundant graph traversals can be further reduced.

While this paper focuses on CFL-reachability-based pointer analysis, the proposed techniques for data sharing and scheduling are expected to be useful for parallelising other graph- 
reachability-based analyses (e.g., bug detection [22], [23]).

In summary, this paper makes the following contributions:

- The first parallel implementation of context- and fieldsensitive pointer analysis with CFL-reachability;

- A data sharing scheme to reduce redundant graph traversals (in all query-processing threads) by graph rewriting;

- A query scheduling scheme to eliminate unnecessary traversals further, by prioritising queries according to their statically estimated dependences; and

- An evaluation with a set of 20 Java benchmarks, showing that our parallel solution achieves an average speedup of 16.2X over a state-of-the-art sequential implementation (with 16 threads) on $16 \mathrm{CPU}$ cores.

The rest of this paper is organised as follows. Section II introduces the intermediate representation used for analysing Java programs and reviews CFL-reachability-based pointer analysis. Section III describes our parallel solution in terms of our data sharing and query scheduling schemes. Section IV evaluates and analyses our solution. Section V discusses the related work. Section VI concludes the paper.

\section{BACKGROUND}

Section II-A describes the intermediate representation used for analysing Java programs. Section II-B reviews the standard formulation of pointer analysis in terms of CFL-reachability.

\section{A. Program Representation}

We focus on Java although this work applies equally well to C [27]. A Java program is represented as a Pointer Assignment Graph (PAG), as defined in Fig. 1.

A node $n$ represents a variable $v$ or an object $o$ in the program, where $v$ can be local $(l)$ or global $(g)$. An edge $e$ represents a statement in the program oriented in the direction of its value flow. An edge connects only to local variables $\left(l_{1}\right.$ and/or $l_{2}$ ) unless it represents an assignment involving at least one global variable $\left(\operatorname{assign}^{g}\right)$. Let us look at the seven types of edges in detail. $l_{1} \stackrel{\text { new }}{\longleftarrow} O$ captures the flow of object $o$ to variable $l_{1}$, indicating that $l_{1}$ points directly to $o . l_{1} \stackrel{\operatorname{assign}^{l}}{\longleftarrow} l_{2}$ represents a local assignment $\left(l_{1}=l_{2}\right)$. A global assignment is similar except that one or both variables at its two sides are static variables in a class (i.e. $g$ ). $l_{1} \stackrel{\operatorname{ld}(\mathrm{f})}{\longleftarrow} l_{2}$ represents a load of the form $l_{1}=l_{2} . f$ and $l_{1} \stackrel{\text { st(f) }}{\longleftarrow} l_{2}$ represents a store of the form $l_{1} . f=l_{2} . l_{1} \stackrel{\text { param }_{i}}{\longleftarrow} l_{2}$ models parameter passing, where $l_{2}$ is an actual parameter and $l_{1}$ is its corresponding formal parameter, at call site $i$. Similarly, $l_{1} \stackrel{\text { ret }_{i}}{\longleftarrow} l_{2}$ indicates an assignment of the return value in $l_{2}$ to $l_{1}$ at call site $i$.

Fig. 2 gives an illustrating example and its PAG representation. Note that $o_{i}$ denotes the object created at the allocation site in line $i$ and $v_{m}$ represents variable $v$ declared in method $m$. Loads and stores to array elements are modeled by collapsing all elements into a special field, denoted arr.

\section{B. CFL-Reachability-based Pointer Analysis}

CFL-reachability [15] is an extension of traditional graph reachability. Let $G$ be a directed graph with edges labelled

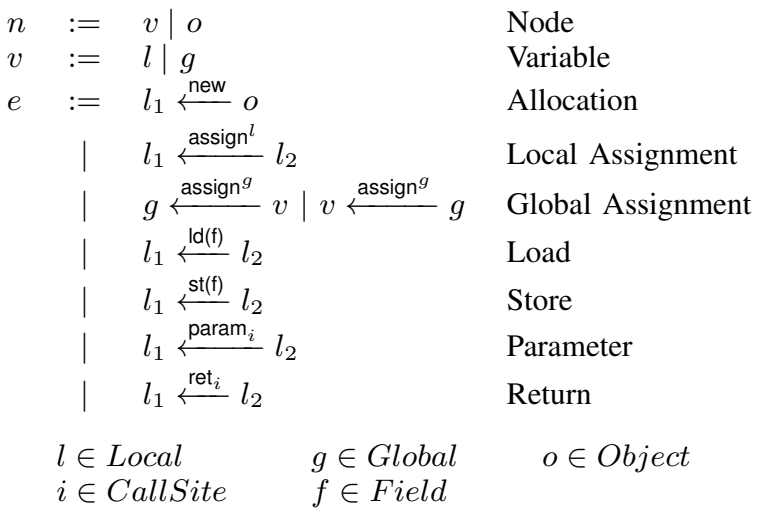

Fig. 1: Syntax of PAG (pointer assignment graph).

by letters over an alphabet $\Sigma$ and $L$ be a CFL over $\Sigma$. Each path $p$ in $G$ is composed of a string $s(p)$ in $\Sigma^{*}$, formed by concatenating in order the labels of edges along $p$. A path $p$ is an L-path iff $s(p) \in L$. Node $x$ is L-reachable to $y$ iff a path $p$ from $x$ to $y$ exists, such that $s(p) \in L$. For a singlesource reachability analysis, the worst-case time complexity is $O\left(\Gamma^{3} N^{3}\right)$, where $\Gamma$ is the size of a normalised grammar for $L$ and $N$ is the number of nodes in $G$.

1) Field-sensitivity: Let us start with a field-sensitive formulation without context-sensitivity. When calling contexts are ignored, there is no need to distinguish the four types of assignments, assign ${ }^{l}, \operatorname{assign}^{g}$, param $_{i}$ and ret $_{i}$; they are all identified as assignment edges of type assign.

For a PAG $G$ with only new and assign edges, the CFL $L_{\mathrm{FT}}$ (FT for flows to) is a regular language, meaning that a variable $v$ is $L_{\mathrm{FT}}$-reachable from an object $o$ in $G$ iff $o$ can flow to $v$. The rule for $L_{\mathrm{FT}}$ is defined as:

$$
\text { flowsTo } \rightarrow \text { new (assign)* }
$$

with flowsTo as the start symbol. If oflowsTo $v$, then $v$ is $L_{\mathrm{FT}^{-}}$ reachable from $o$. For example, in Fig. 2(b), since $\mathrm{O}_{15} \stackrel{\text { new }}{\longrightarrow}$ $\mathrm{v} 1_{\text {main }} \stackrel{\text { param }_{15}}{\longrightarrow}$ this vector, $\mathrm{O}_{15}$ flows to this vector.

When field accesses are also handled, the CFL $L_{\mathrm{FS}}$ (FS for field-sensitivity) is defined as follows:

$$
\begin{aligned}
\text { flowsTo } & \rightarrow \text { new }(\text { assign } \mid \mathrm{st}(\mathrm{f}) \text { alias } \mathrm{ld}(\mathrm{f}))^{*} \\
\text { alias } & \rightarrow \overline{\text { flowsTo } \text { flowsTo }} \\
\overline{\text { flowsTo }} & \rightarrow(\overline{\text { assign }} \mid \overline{\operatorname{ld}(\mathrm{f})} \text { alias } \overline{\mathrm{st}(\mathrm{f})})^{*} \overline{\text { new }}
\end{aligned}
$$

The rule for flowsTo matches fields via st(f) alias $\mathrm{ld}(\mathrm{f})$, where $s t(f)$ and $I d(f)$ are like a pair of parentheses [19]. For a pair of statements $q . f=y(\operatorname{st}(f))$ and $x=p . f(\operatorname{ld}(f))$, if $p$ and $q$ are aliases, then an object $o$ that flows into $y$ can flow first through this pair of parentheses (i.e. st $(f)$ and $\operatorname{ld}(f))$ and then into $x$. For example, in Fig. 2(b), as $\mathrm{o}_{15} \stackrel{\text { new }}{\longrightarrow}$ $\mathrm{v} 1_{\text {main }} \stackrel{\text { param }_{15}}{\longrightarrow}$ this Vector $_{\text {and }} \mathrm{O}_{15} \stackrel{\text { new }}{\longrightarrow} \mathrm{v} 1_{\text {main }} \stackrel{\text { param } 18}{\longrightarrow}$ this get $_{\text {get }}$, we have thisvector alias thisget. Thus $0_{6} \stackrel{\text { new }}{\longrightarrow} t_{\text {vector }} \stackrel{\text { st(elems) }}{\longrightarrow}$ thisvector alias this get $^{\text {Id(elems) }} \longrightarrow t_{\text {get }}$, indicating that $\mathrm{O}_{6}$ flows to $\mathrm{t}_{\text {get }}$.

To allow the alias relation in the language, $\overline{\text { flowsTo }}$ is introduced as the inverse of the flowsTo relation. For a flowsTopath $p$, its corresponding $\overline{\text { flowsTo }}$-path $\bar{p}$ can be obtained using 


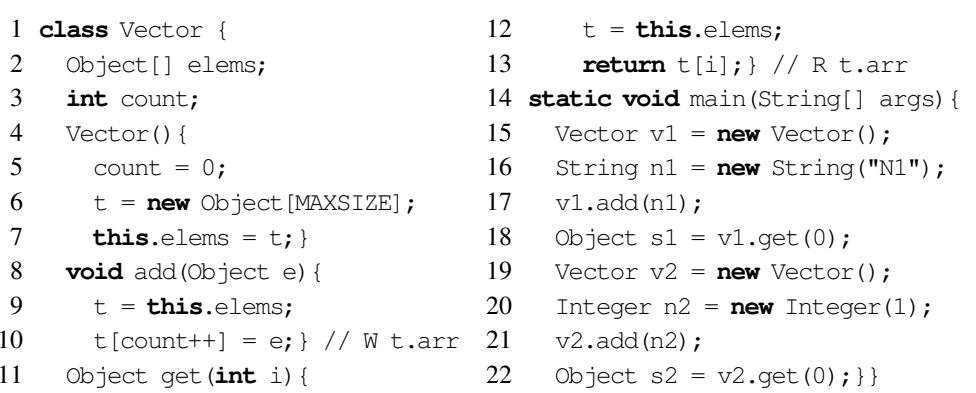

(a) Java code

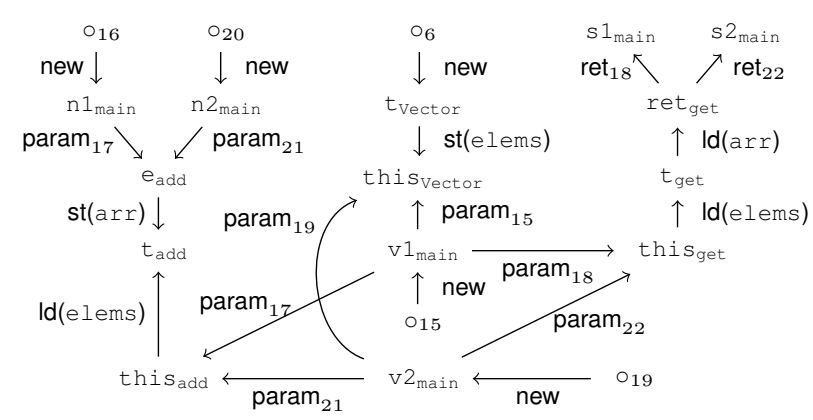

(b) PAG

Fig. 2: A Java example and its PAG.

inverse edges, and vice versa. For each edge $x \stackrel{e}{\leftarrow} y$ in $p$, its inverse edge is $y \stackrel{\bar{e}}{\leftarrow} x$ in $\bar{p}$. Therefore, o flowsTo $x$ iff

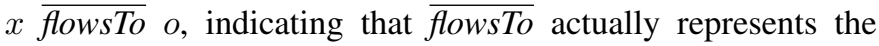
points-to relation. To find the points-to set of a variable, we use the CFL given in (2) with $\overline{\text { flowsTo }}$ as the start symbol.

2) Context-sensitivity: When context-sensitivity is considered, param ${ }_{i}$ and ret ${ }_{i}$ are treated as assign as before in $L_{\mathrm{FS}}$. However, assign ${ }^{l}$ and assign ${ }^{g}$ are now distinguished.

A context-sensitive CFL requires param ${ }_{i}$ and ret $_{i}$ to be matched, also in terms of balanced parentheses, by ruling out the unrealisable paths in a program [18]. The CFL $R_{\mathrm{CS}}(\mathrm{CS}$ for context-sensitivity) for capturing realisable paths is:

$$
\begin{aligned}
& c \rightarrow \text { entry }_{i} c \text { exit }_{i}|c c| \epsilon \\
& \text { entry }_{i} \rightarrow \operatorname{param}_{i} \mid \overline{\text { ret }}_{i} \\
& \text { exit }_{i} \rightarrow \operatorname{ret}_{i} \mid \overline{\text { param }}_{i}
\end{aligned}
$$

When traversing along a flowsTo path, after entering a method via param $_{i}$ from call site $i$, a context-sensitive analysis requires exiting from that method back to call site $i$, via either ret ${ }_{i}$ to continue its traversal along the same flowsTo path or $\overline{\text { param }}$ i to start a new search for a flowsTo path. Traversing along a flowsTo path is similar except that the direction of traversal is reversed. Consider Fig. 2(b). $s 1_{\text {main }}$ is found to point to $O_{16}$ as $O_{16}$ reaches $s 1_{\text {main }}$ along a realisable path by first matching param ${ }_{17}$ and $\overline{\text { param }_{17}}$ and then param 18 and ret $t_{18}$. However, $s 1_{\text {main }}$ does not point to $O_{20}$ since $\mathrm{O}_{20}$ cannot reach $\mathrm{s} 1_{\text {main }}$ along a realisable path.

Let $L_{\mathrm{PT}}$ (PT for points-to) be the CFL for computing the points-to information of a variable field- and contextsensitively. Then $L_{\mathrm{PT}}$ is defined in terms of (2) and (3): $L_{\mathrm{PT}}=L_{\mathrm{FS}} \cap R_{\mathrm{CS}}$, where $\overline{\text { flowsTo }}$ is the start symbol.

3) Algorithm: With CFL-reachability, a query that requests for a variable's points-to information can be answered ondemand, according to Algorithm 1. This algorithm makes use of three variables: (1) $E$ represents the edge set of the PAG of the program, (2) $B$ is the (global) budget defined as the maximum number of steps that can be traversed by any query, with each node traversal being counted as one step [19], and (3) steps is query-local, representing the number of steps that has been traversed so far by a particular query.

Given a query $(l, c)$, where $l$ is a local variable and $c$ is

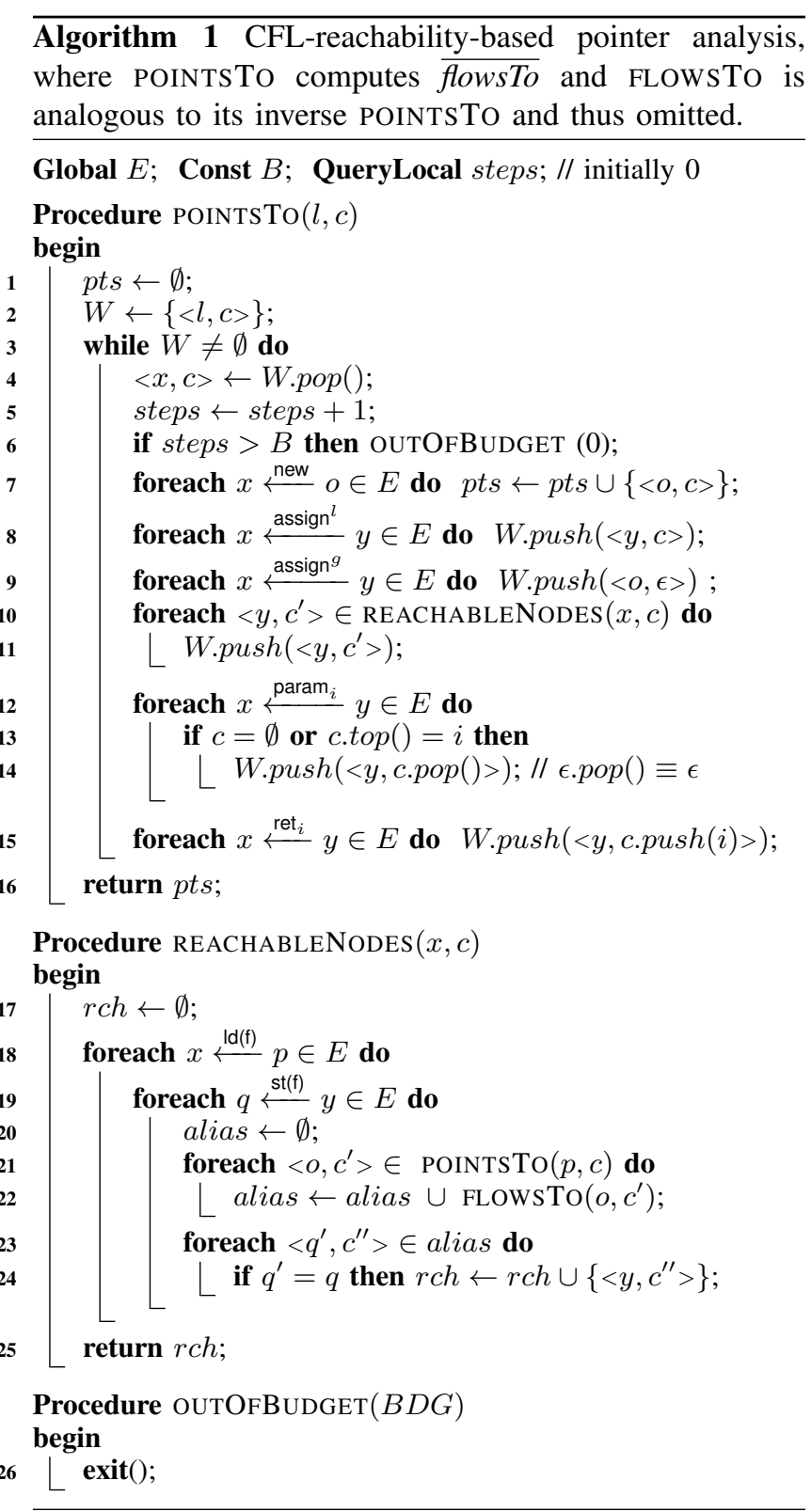


a context, POINTSTO computes the points-to set of $l$ under $c$. It traverses the PAG with a work list $W$ maintained for variables to be explored. $p t s$ is initialised with an empty set and $W$ with $\langle l, c\rangle$ (lines $1-2$ ). By default, steps for this query is initialised as 0 . Each variable $x$ with its context $c$, i.e., $\langle x, c\rangle$ obtained from $W$ is processed as follows: steps is updated, triggering a call to OUTOFBUDGET if the remaining budget is 0 (lines $5-6$ ), and the incoming edges of $x$ are traversed according to (2) and (3) (lines $7-15$ ).

Field-sensitivity is handled by $\operatorname{REACHABLENOdes}(x, c)$, which searches for the reachable variables $y$ to $x$ in context $c$, due to heap accesses by matching the load $(x=p . f)$ with every store $(q . f=y)$, where $p$ and $q$ are aliases (lines 17 $25)$. Both POINTSTO and FLOWSTO are called (recursively) to ensure that $p$ and $q$ are aliased base variables.

To handle context-sensitivity, the analysis stays in the same context $c$ for assign ${ }^{l}$ (line 8), clears $c$ for assign ${ }^{g}$ as global variables are treated context-insensitively (line 9), matches the context $\left(c . t o p()=i\right.$ ) for param $_{i}$ but allows for partially balanced parentheses when $c=\emptyset$ since a realizable path may not start and end in the same method (lines 12 - 14), and pushes call site $i$ into context $c$ for ret $_{i}$ (line 15).

\section{Methodology}

CFL-reachability-based pointer analysis is driven by queries issued by application clients. There are two main approaches to dividing work among threads, based on different levels of parallelism available: intra-query and inter-query.

To exploit intra-query parallelism, we need to partition and distribute the work performed in computing the points-to set of a single query among different threads. Such parallelism is irregular and hard to achieve with the right granularity. In addition, considerable synchronisation overhead that may be incurred would likely offset the performance benefit achieved.

To exploit inter-query parallelism, we assign different queries to different threads, harnessing modern multicore processors. This makes it possible to obtain parallelism without incurring synchronisation overhead unduly. In addition, some clients may issue queries in batch mode for a program. For example, the points-to information may be requested for all variables in a method, a class, a package or even the entire program. This provides a further optimisation opportunity. The focus of this work is on exploiting inter-query parallelism.

\section{A. A Naive Parallelisation Strategy}

A naive approach to exploiting inter-query parallelism is to maintain a lock-protected shared work list for queries and let each thread fetch queries (to process) from the work list until the work list is empty. While achieving some good speedups (over the sequential setting), this naive strategy is inefficient due to a large number of redundant graph traversals made. We propose two schemes to reduce such redundancies. Section III-B describes our data sharing scheme, while Section III-C explains our query scheduling scheme.

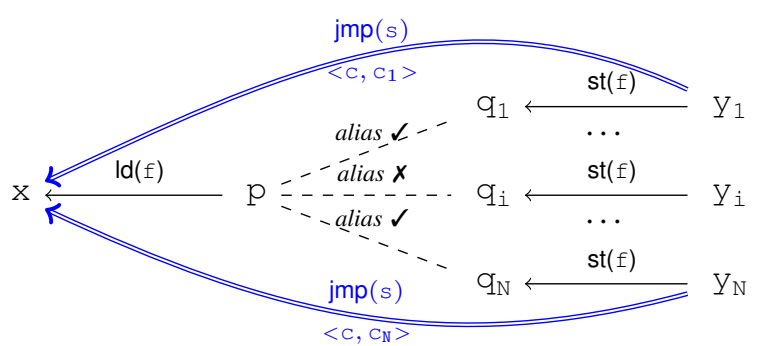

(a) Within budget: all $N$ stores analysed completely in $s$ steps from $(x, c)$

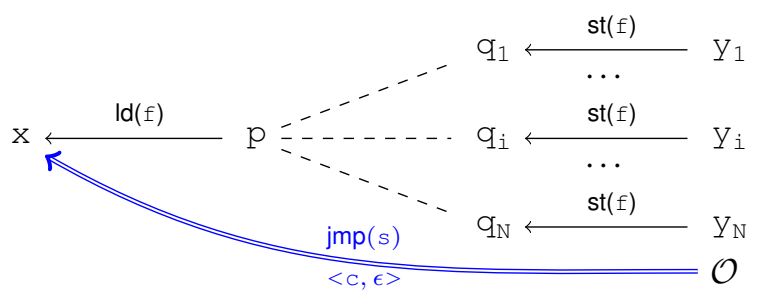

(b) Out of budget: fewer than $N$ stores analysed in $s$ steps from $(x, c)$

Fig. 3: Adding jmp edges by graph rewriting, for a single iteration of the loop in line 18 of $\operatorname{REACHABLENODES}(x, c)$. In (a), $x \frac{\operatorname{jmp}(s)}{\left.<c, c_{k}\right\rangle} y_{k}$ is introduced for each $\left(y_{k}, c_{k}\right)$ added to $r c h$ in line 24 of REACHABLENodes $(x, c)$ when $p$ and $q_{k}$

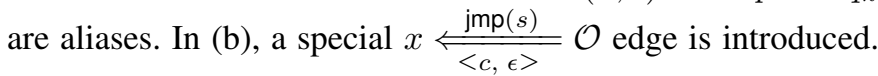

\section{B. Data Sharing}

Given a program, we are motivated to add edges to its PAG to serve as shortcuts for some paths traversed in a query so that subsequent queries may take the shortcuts instead of retraversing their associated paths (redundantly). The challenge here is to perform data sharing context- and field-sensitively. Section III-B1 formulates data sharing in terms of graph rewriting. Section III-B2 gives an algorithm for realising data sharing in the CFL-reachability framework.

1) Data Sharing by Graph Rewriting: We choose to share paths involving heap accesses, which tend to be long (timeconsuming to traverse) and common (repeatedly traversed across the queries). As illustrated in Fig. 3, we do so by avoiding making redundant alias tests in $\operatorname{REACHABLENODES}(x, c)$. For its loop at line 18 , each iteration starts with a load $x=p . f$ and then examines all the $N$ matching stores $q_{1} . f=$ $y_{1}, \ldots, q_{N} \cdot f=y_{N}$ at line 19. For each $q_{k} . f=y_{k}$ accessed in context $c_{k}$ such that $q_{k}$ is an alias of $p,\left(y_{k}, c_{k}\right)$ is inserted into $r c h$, meaning that $(x, c)$ is reachable from $\left(y_{k}, c_{k}\right)$ (lines 20 - 24). Note that during this process, mutually recursive calls to pointsto(), Flowsto() and Reachablenodes() for discovering other aliases are often made.

There are two cases due to the budget constraint. Fig. 3(a) illustrates the case when an iteration of line 18 is completely analysed in $s$ steps starting from $(x, c)$ within the pre-set budget. A jmp edge, $x \stackrel{\text { jmp }(s)}{\stackrel{<c, c_{k}>}{\text { s }}} y_{k}$, is added for each $q_{k}$ that is an alias of $p$. Instead of rediscovering the path from 
$(x, c)$ to $\left(y_{k}, c_{k}\right)$, a subsequent query will take this shortcut.

Fig. 3(b) explains the other case when an iteration of line 18 is only partially analysed since the analysis runs out of budget after $s$ steps have elapsed from $(x, c)$. A special jmp edge,

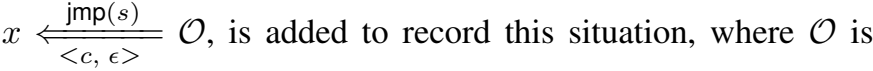
a special node added and $\epsilon$ is a "don't-care" context. A later query will benefit from this special shortcut by making an early termination (ET) if its remaining budget is smaller than $s$.

Therefore, we have formulated data sharing as a graph rewriting problem by adding jmp edges to the PAG of a program, in terms of the syntax given in Fig. 4.

$$
\begin{aligned}
& l:=\ldots \mid \mathcal{O} \quad \text { Extended Local Variable } \\
& e:=\ldots \\
& \text { | } \quad l_{1} \underset{\operatorname{jmp}(s)}{\Longleftarrow c_{1}, c_{2}>} l_{2} \quad \text { Jump (or Shortcut) } \\
& \mathcal{O} \text { is Unfinished } \quad c_{1}, c_{2} \in \text { Context }
\end{aligned}
$$

Fig. 4: Syntax of extended PAG.

As described below, jmp edges are added on the fly during the analysis. Given a PAG extended with such jmp edges, the CFL given earlier in (2) is modified to:

$$
\begin{aligned}
\text { flowsTo } & \rightarrow \text { new }(\text { assign }|\operatorname{jmp}(s)| \operatorname{st}(\mathrm{f}) \text { alias } \mathrm{ld}(\mathrm{f}))^{*} \\
\text { alias } & \rightarrow \overline{\text { flowsTo flowsTo }} \\
\overline{\text { flowsTo }} & \rightarrow(\overline{\text { assign }}|\overline{\operatorname{jmp}(s)}| \overline{\operatorname{ld}(\mathrm{f})} \text { alias } \overline{\mathrm{st}(\mathrm{f})})^{*} \overline{\text { new }}
\end{aligned}
$$

By definition of jmp, this modified CFL generates the same language as the original CFL if all jmp edges of the type illustrated in Fig. 3(b) (for handling OUTOFBUDGET) in the PAG of a program are ignored, since the jmp edges of the other type illustrated in Fig. 3(a) serve as shortcuts only. Two types of jmp edges are exploited in our parallel implementation to accelerate its performance as described below.

2) Algorithm: With data sharing, REACHABLENodes $(x, c)$ in Algorithm 1 is revised as shown in Algorithm 2. There are three cases, the original one plus the two shown in Fig. 3:

- In the if branch (line 2) for handling the scenario depicted in Fig. 3(b), the analysis makes an early termination by calling REACHABLENODES() if its remaining budget at $(x, c), B-$ steps, is smaller than $s$. Otherwise, the analysis moves to execute the second else.

- In the first else branch for handling the scenario in Fig. 3(a), the analysis takes the shortcuts identified by the $\operatorname{jmp}(s)$ edges instead of re-traversing its associated paths. The same precision is maintained even if we do not check the remaining budget $B$-steps against $s$, since the source node of a jmp edge is a variable (not an object). When this variable is explored later, the remaining budget will be checked in line 6 of Algorithm 1 or line 3 of Algorithm 2.

- In the second else branch, we proceed as in REACHABleNodes $(x, c)$ given in Algorithm 1 except that we will need to add the jmp edge(s) as illustrated in

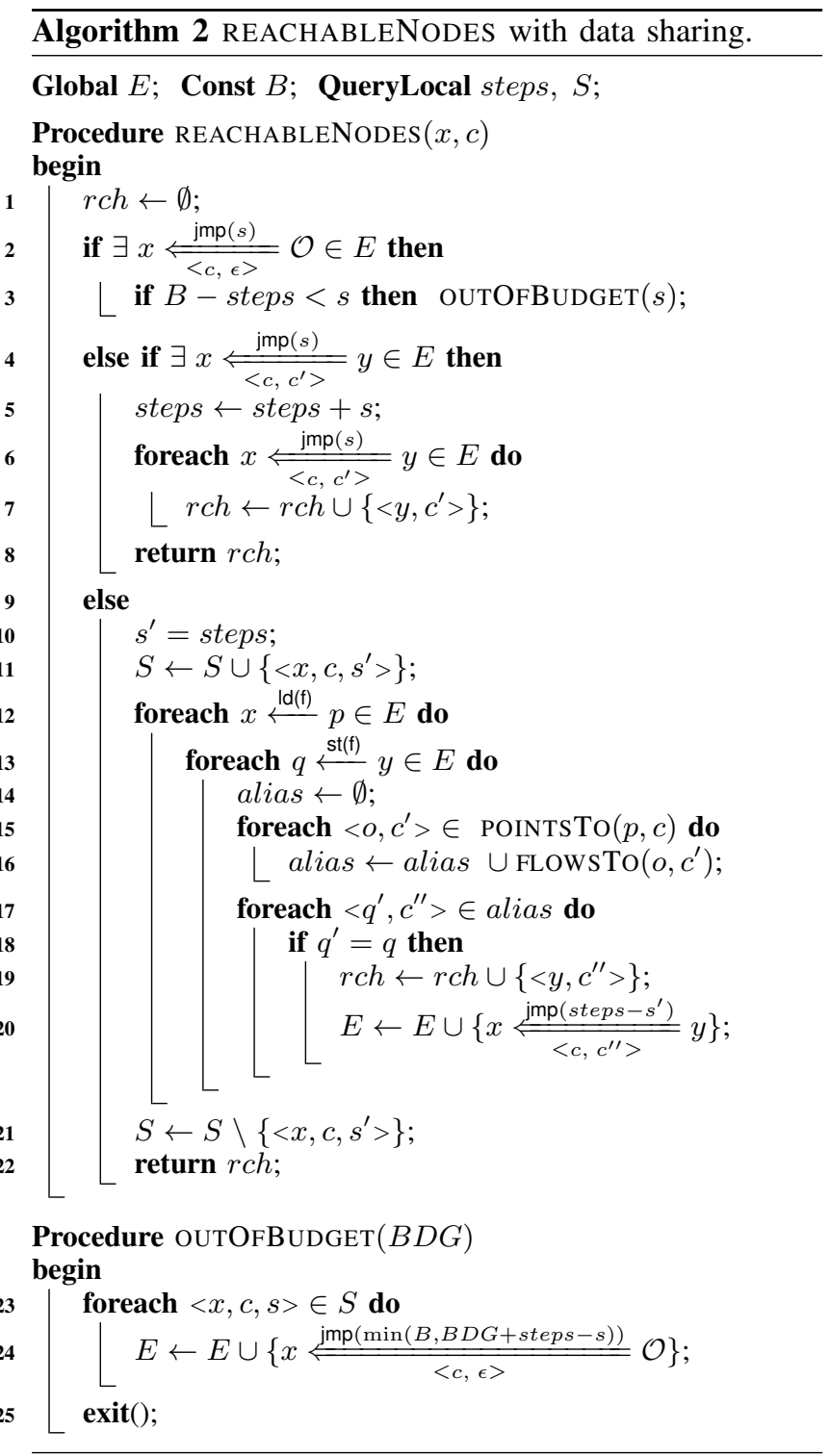

either Fig. 3(a) (line 20) or Fig. 3(b) (line 24).

OutOFBudget $(B D G)$ is called from line 6 (by passing 0 ) in Algorithm 1 or line 3 in Algorithm 2 (by passing $s$ ). In both cases, let $n$ be the node visited before the call. With a remaining budget no larger than $B D G$ on encountering $n$, the analysis will surely run out of budget eventually. For each $(x, c, s) \in S$, the analysis first reaches $(x, c)$ and then $n$ in steps $-s$ steps. Thus, $x \frac{\operatorname{jmp}(\min (B, B D G+\text { steps }-s))}{\langle c, \epsilon>} \mathcal{O}$ is added.

\section{Query Scheduling}

The order in which queries are processed affects the number of early terminations made, due to $B-$ steps $<s$ tested in line 3 of Algorithm 2, where $s$ appears in a $\operatorname{jmp}(s)$ edge that was added in an earlier query and steps is the number of steps already consumed by the current query. In general, if we handle a variable $y$ before those variables $x$ such that $x$ is reachable from $y$, then more early terminations may result. 
To increase early terminations, we organise queries (available in batch mode) in groups and assign a group of queries rather than a single query to a thread at a time to reduce synchronisation overhead on the shared work list for queries. We discuss below how the queries in a group and the groups themselves are scheduled. Section III-C1 describes how to group queries. Section III-C2 discusses how to order queries. Section III-C3 gives an illustrating example.

1) Grouping Queries: A group contains all possible variables such that every member is connected with at least another member in the PAG of the program via the following relation:

$$
\text { direct } \rightarrow\left(\operatorname{assign}^{l}\left|\operatorname{assign}^{g}\right| \operatorname{param}_{i} \mid \operatorname{ret}_{i}\right)^{*}
$$

Both $l_{1} \stackrel{\operatorname{ld}(f)}{\longleftarrow} l_{2}$ and $l_{1} \stackrel{\text { st(f) }}{\longleftarrow} l_{2}$ edges are not included since there is no reachability between $l_{1}$ and $l_{2}$.

2) Ordering Queries: For the variables in the same group, we use their so-called connection distances $(C D s)$ to determine their issuing order. The $C D$ of a variable in a group is defined as the length of the longest path that contains the variable in the group (modulo recursion). For the variables in a group, the shorter their $C D$ s are, the earlier they are processed.

For different groups, we use their so-called dependence depths $(D D s)$ to determine their scheduling order. For example, computing POINTSTo $(x, c)$ for $x$ in Algorithm 1 depends on the points-to set of the base variable $p$ in load $x=p . f$ (line 21). Preferably, $p$ should be processed earlier than $x$.

To quantify the $D D$ of a group, we estimate the dependences between variables based on their (static) types. We define the level of a type $t$ (with respect to its containment hierarchy) as:

$$
L(t)= \begin{cases}\max _{t_{i} \in F_{T}(t)} L\left(t_{i}\right)+1 & \text { isRef }(t) \\ 0 & \text { otherwise }\end{cases}
$$

where $F_{T}(t)$ enumerates the types of all instance fields of $t$ (modulo recursion) and is $\operatorname{Ref}(t)$ is true if $t$ is a reference type. The $D D$ of a variable of type $t$ is defined to be $1 / L(t)$. Note that the DD of a static variable is also approximated heuristically this way. The DD of a group of variables is defined as the smallest of the DDs of all variables in the group.

During the analysis, groups are issued (sorted) in increasing values of their DDs. Let $M$ be the average size of these groups. To ensure load balance, groups larger than $M$ are split and groups smaller than $M$ are merged with their adjacent groups, so that each resulting group has roughly $M$ variables.

3) An Example: In Fig. 5, we focus on its three variables $x, y$, and $z$, which are assumed to all run out of budget $B$. According to (5), as shown in Fig. 5(a), $x$ and $y$ (together with $w$ ) are in one group and $z$ (together with $p$ ) is in another group. The $C D$ s of $x, y$ and $z$ are 100, 200 and 300 steps, respectively. As both $x$ and $y$ depend on $z$, the latter group will be scheduled before the former group. As a result, our query scheduling scheme will likely cause $x, y$ and $z$ to be processed sequentially according to $\mathrm{O}_{3}$ (in some thread interleaving) among the three orders, $O_{1}, O_{2}$ and $O_{3}$, listed in Fig. 5(b).

For $O_{1}, y$ is processed first, which takes $B$ steps (i.e., the

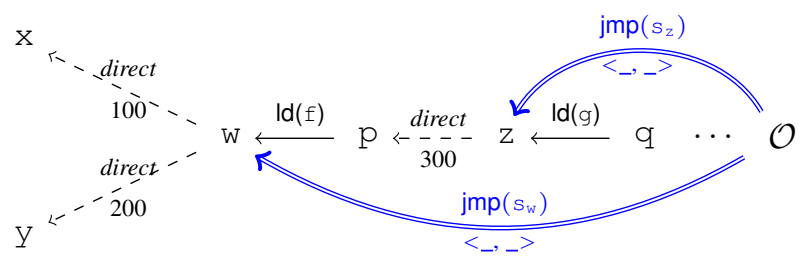

(a) PAG with the direct relation

\begin{tabular}{|c|ccc|cc|c|}
\hline \multirow{2}{*}{ Order } & \multicolumn{2}{|c|}{ Traversed \#Steps } & \multicolumn{2}{|c|}{$j \mathrm{jmp}(s)$} & \multirow{2}{*}{ \#ETs } \\
\cline { 2 - 6 } & $x$ & $y$ & $z$ & $s_{z}$ & $s_{w}$ & \\
\hline \hline$O_{1}: y, x, z$ & $B$ & $B$ & $B$ & $B-500$ & $B-200$ & 0 \\
$O_{2}: x, y, z$ & $B$ & 200 & $B$ & $B-400$ & $B-100$ & 1 \\
$O_{3}: z, x, y$ & 400 & 200 & $B$ & $B$ & $B$ & 2 \\
\hline
\end{tabular}

(b) Three scheduling orders

Fig. 5: An example of query scheduling, where $x$ has a smaller $\mathrm{CD}$ than $y$ and $\{x, y\}$ has a higher DD than $\{z\}$.

maximum budget allowed), with the two jmp edges added as shown, where $s_{z}=B-500$ and $s_{w}=B-200$. When $x$ is processed next, neither shortcut will be taken, since $x$ still has more budget remaining: $B-400>B-500$ at $z$ and $B-100>B-200$ at $w$. Similarly, the two shortcuts do not benefit $z$ either. Thus, no early termination occurs.

For $O_{2}, \mathrm{x}$ is issued first, resulting in also the same two jmp edges added, except that $s_{z}=B-400$ and $s_{w}=B-100$. So when $y$ is handled next, an early termination is made at $w$, since its budget remaining at $w$ is $B-200\left(<s_{w}=B-100\right)$.

According to $\mathrm{O}_{3}$, the order that is mostly likely induced by our query scheduling scheme, $z$ is processed first. Only the $\operatorname{jmp}\left(s_{z}\right)$ edge at $z$ is added, where $s_{z}=B$. When $\mathrm{x}$ is analysed next, $z$ is reached in 400 steps. Taking $\operatorname{jmp}\left(s_{z}\right)$ (since $\left.B-400<s_{z}=B\right)$, an early termination is made. Meanwhile, the $\operatorname{jmp}\left(s_{w}\right)$ edge at $w$ is added, where $s_{w}=B$. Finally, $y$ is issued, causing $w$ to be visited in 200 steps. Taking $\operatorname{jmp}\left(s_{w}\right)$ (since $B-200<s_{w}=B$ ), another early termination is made. Of the three orders illustrated in Fig. 5(b), $O_{3}$ is likely to cause more early terminations, resulting in fewer traversal steps.

\section{Evaluation}

We demonstrate that our parallel implementation of CFL-reachability-based pointer analysis achieves significant speedups than a state-of-the-art sequential implementation.

\section{A. Implementations}

The sequential one is coded in Java based on the publicly available source-code implementation of the CFL-reachabilitybased pointer analysis [18] in Soot 2.5.0 [24], with its nonrefinement (general-purpose) configuration used. Note that the refinement-based configuration is not well-suited to certain clients such as null-pointer detection. Our parallel implementation given in Algorithms 1 and 2 are also coded in Java. In both cases, the per-query budget $B$ is set as 75,000 steps, recursion cycles of the call graph are collapsed, and points-to cycles are eliminated as described as in [18]. 


\begin{tabular}{|c|c|c|c|c|c|c|c|c|c|c|c|c|}
\hline Benchmark & \#Classes & \#Methods & \#Nodes & \#Edges & \#Queries & $T_{S e q}(\mathrm{secs})$ & \#Jumps & $\# S\left(\times 10^{6}\right)$ & $R_{S}$ & $\overline{S_{g}}$ & $\# E T s$ & $R_{E T}$ \\
\hline _200_check & $\overline{5,758}$ & 54,514 & 225,797 & 429,551 & 1,101 & 2.88 & 428 & 4.14 & 25.76 & 16.7 & $\overline{0}$ & $\overline{1}$ \\
\hline _201_compress & 5,761 & 54,549 & 225,765 & 429,808 & 1,328 & 3.72 & 1,210 & 4.21 & 8.42 & 4.6 & 5 & 1.00 \\
\hline _202_jess & 5,901 & 55,200 & 232,242 & 440,890 & 7,573 & 121.11 & 4,755 & 193.77 & 42.68 & 16.1 & 617 & 1.15 \\
\hline 205_raytrace & 5,774 & 54,681 & 227,514 & 432,110 & 3,240 & 9.39 & 2,325 & 62.02 & 92.84 & 7.2 & 8 & 0.88 \\
\hline $209 \mathrm{db}$ & 5,753 & 54,549 & 225,994 & 430,569 & 1,339 & 16.98 & 4,202 & 10.06 & 10.02 & 10.3 & 18 & 1.17 \\
\hline 213_javac & 5,921 & 55,685 & 240,406 & 473,680 & 14,689 & 258.34 & 5,309 & 467.28 & 64.60 & 9.2 & 76 & 0.99 \\
\hline _222_mpegaudio & 5,801 & 54,826 & 230,349 & 435,391 & 6,389 & 46.52 & 2,306 & 86.17 & 53.33 & 3.8 & 53 & 3.17 \\
\hline _227_mtrt & 5,774 & 54,681 & 227,514 & 432,110 & 3,241 & 10.38 & 2,358 & 62.17 & 115.70 & 7.2 & 7 & 0.86 \\
\hline $228 \_$jack & 5,806 & 54,830 & 229,482 & 435,159 & 6,591 & 39.54 & 25,030 & 79.48 & 40.03 & 14.2 & 100 & 1.62 \\
\hline _999_checkit & 5,757 & 54,548 & 226,292 & 431,435 & 1,473 & 12.61 & 2,180 & 10.14 & 7.94 & 16.9 & 23 & 0.78 \\
\hline avrora & 3,521 & 29,542 & 108,210 & 189,081 & 24,455 & 51.16 & 32,046 & 47.46 & 6.18 & 9.4 & 24 & 2.83 \\
\hline batik & 7,546 & 65,899 & 252,590 & 477,113 & 64,467 & 72.72 & 14,876 & 114.57 & 11.95 & 10.3 & 38 & 1.37 \\
\hline fop & 8,965 & 79,776 & 266,514 & 636,776 & 71,542 & 118.22 & 25,418 & 169.92 & 19.03 & 18.6 & 76 & 1.20 \\
\hline h2 & 3,381 & 32,691 & 115,249 & 204,516 & 44,901 & 25.50 & 22,094 & 91.38 & 12.39 & 16.0 & 283 & 0.66 \\
\hline luindex & 3,160 & 28,791 & 108,827 & 191,126 & 22,415 & 23.28 & 62,457 & 60.93 & 8.72 & 8.2 & 113 & 0.71 \\
\hline lusearch & 3,120 & 28,223 & 109,439 & 193,012 & 17,520 & 57.78 & 77,153 & 66.26 & 7.90 & 9.3 & 75 & 1.52 \\
\hline pmd & 3,786 & 33,432 & 110,388 & 195,834 & 56,833 & 61.05 & 77,313 & 69.10 & 7.93 & 9.2 & 84 & 1.06 \\
\hline sunflow & 6,066 & 56,673 & 233,459 & 447,002 & 21,339 & 55.56 & 20,946 & 49.04 & 5.57 & 7.4 & 24 & 2.38 \\
\hline tomcat & 8,458 & 83,092 & 265,015 & 574,236 & 185,810 & 202.89 & 24,601 & 243.90 & 23.14 & 13.1 & 574 & 1.33 \\
\hline xalan & 3,716 & 33,248 & 109,317 & 192,441 & 56,229 & 54.11 & 33,459 & 60.35 & 7.90 & 9.4 & 82 & 1.43 \\
\hline Average & 5,486 & 50,972 & 198,518 & 383,592 & 30,624 & 62.19 & 22,023 & 97.62 & 28.6 & 10.9 & 114.0 & 1.35 \\
\hline
\end{tabular}

TABLE I: Benchmark information and statistics.

In our parallel implementation, we use a Concurrent HashMap to manage jmp edges efficiently. We apply a simple optimisation to further reduce synchronisation incurred and thus achieve better speedups.

If we create jmp edges exhaustively for all the paths discovered in Algorithm 2, the overhead incurred by such operations as search, insertion and synchronisation on the map may outweigh the performance benefit obtained. As an optimisation, we will introduce the $\operatorname{jmp}(s)$ edges in Fig. 3(a) only when $s \geq \tau_{F}$ and the special $\operatorname{jmp}(s)$ edge in Fig. 3(b) only when $s \geq \tau_{U}$, where $\tau_{F}$ and $\tau_{U}$ are tunable parameters. In our experiments, we set $\tau_{F}=100$ and $\tau_{U}=10000$. Their performance impacts are evaluated in Section IV-D2.

For the case in Fig. 3(a), the set of all jmp edges is associated with the key $(x, c)$ when inserted into the map. So no two threads reaching $(x, c)$ simultaneously will insert this set of jmp edges twice into the map. For the case in Fig. 3(b),

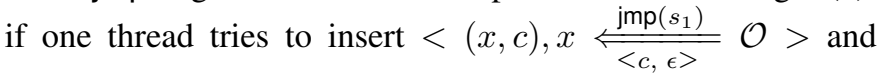

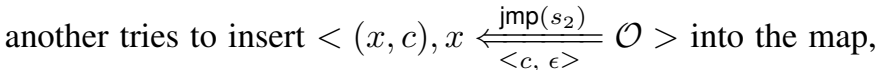
only one of the two will succeed. An attempt that selects the one with the large $s$ value (to increase early terminations) can be cost-ineffective due to the extra work performed.

\section{B. Experimental Setting}

The multi-core system used in our experiments is equipped with two Intel Xeon E5-2650 CPUs with 62GB of RAM. Each CPU has 8 cores, which share a unified 20MB L3 cache. Each CPU core has a frequency of $2.00 \mathrm{MHz}$, with its own $\mathrm{L} 1$ cache of $64 \mathrm{~KB}$ and L2 cache of $256 \mathrm{~KB}$. The Java Virtual Machine used is the Java HotSpot 64-Bit Server VM (version 1.7.0_40), running on a 64-bit Ubuntu 12.04 operating system.

\section{Methodology}

We evaluate the performance advantages of our parallel implementation over the sequential one by comparing the query-processing times taken in both cases. SEQCFL denotes the sequential implementation. In order to assess the effectiveness of our parallel implementation, we consider a number of variations. PARCFL ${ }_{m}^{t}$ represents a particular parallel implementation, where $t$ stands for the number of threads used. Here, $m$ indicates one of the three parallelisation strategies used: (1) the naive solution described in Section III-A when $m=$ naive, (2) our parallel solution with the data sharing scheme described in Section III-B enabled when $m=D$, and (3) the parallel solution (2) with the query scheduling scheme described in Section III-C also enabled when $m=D Q$.

Table I lists a set of 20 Java benchmarks used, consisting of all the 10 SPEC JVM98 benchmarks and 10 additional benchmarks from the DaCapo 2009 benchmark suite. For each benchmark, the analysed code includes both the application code and the related library code, with their class and method counts given in Columns 2 and 3, respectively. The node and edge counts in the original PAG of a benchmark are given in Columns 4 and 5, respectively. For each benchmark, the queries that request points-to information are issued for all the local variables in its application code, collected from Soot 2.5.0 as in prior work [17], [25]. Note that more queries are generated in some DaCapo benchmarks than some JVM98 benchmarks even though the DaCapo benchmarks have smaller PAGs. This is because the JVM98 benchmarks involve more library code. The remaining columns are explained below.

\section{Performance Results}

We examine the performance benefits of our parallel pointer analysis and the causes for the speedups obtained.

1) Speedups: Fig. 6 shows the speedups of our parallel implementation over SEQCFL (as the baseline), where the analysis times of SEQCFL for all the benchmarks are given in Column 7 of Table I. Note that SEQCFL is $49 \%$ faster than the open-source sequential implementation of [18] in Soot 2.5.0, since we have simplified some of its heuristics and em- 


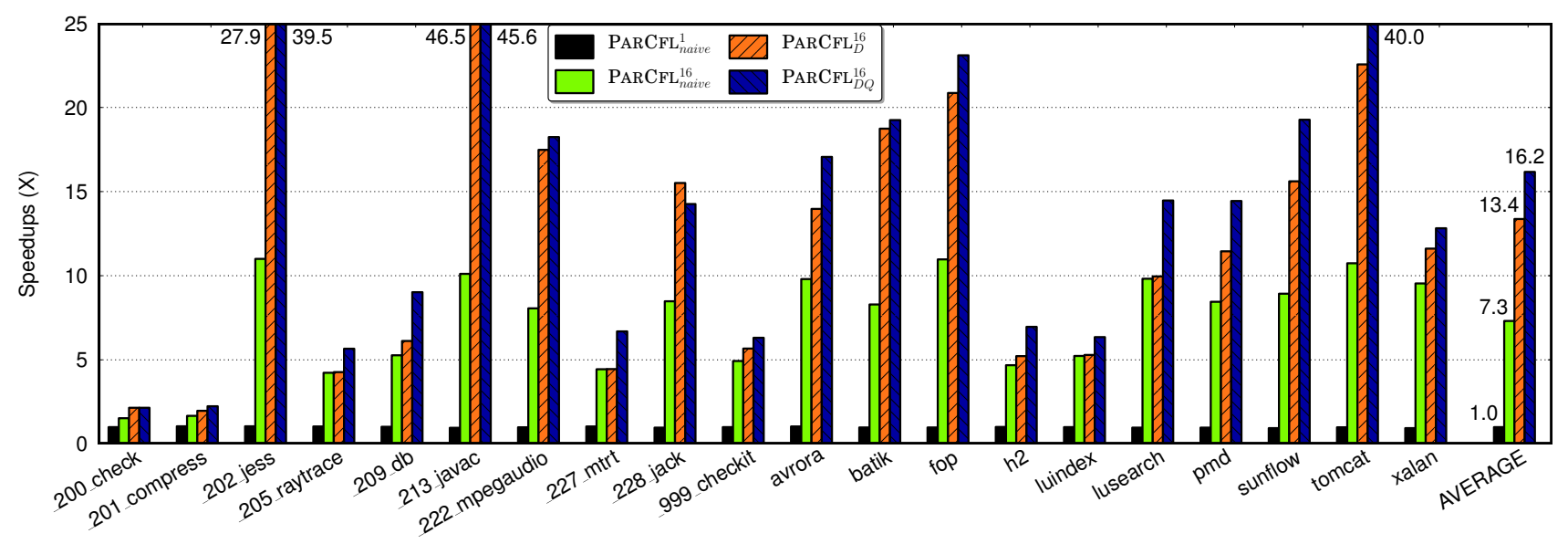

Fig. 6: Speedups of our parallel implementation (in various configurations) normalised with respect to SEQCFL.

ployed different data structures. When the naive parallelisation strategy is used, PARCFL ${ }_{\text {naive }}^{1}$ (with one single thread) is as efficient as SEQCFL, since the locking overhead incurred for retrieving the queries from the shared work list is negligible. With 16 threads, PARCFL ${ }_{\text {naive }}^{16}$ attains an average speedup of 7.3X. When our data sharing scheme is used, PARCFL $_{D}^{16}$ runs a lot faster, with the average speedup being pushed up further to $13.4 \mathrm{X}$. When our query scheduling scheme is also enabled, PARCFL $_{D Q}^{16}$, which traverses significantly fewer steps than SEQCFL, has finally reached an average speedup of $16.2 X$. The superlinear speedups are achieved in some benchmarks due to the avoidance of redundant traversals (a form of caching) in all concurrent query-processing threads as analysed below.

2) Effectiveness of Data Sharing: Our data sharing scheme, which enables the traversal information obtained in a query to be shared by subsequent queries via graph rewriting, has succeeded in accelerating the analysis on top of the naive parallelisation strategy (PARCFL $\mathrm{Paive}^{t}$ ) for all benchmarks.

To understand its effectiveness, some statistics are given in Columns $8-10$ in Table I. For a benchmark, \#Jumps denotes the number of jmp edges added to its PAG due to data sharing, \#S represents the total number of steps traversed by SEQCFL (without data sharing) for all the queries issued from the benchmark, and $R_{S}$ is the ratio of the number of steps saved by the jmp edges for the benchmark over the number of steps traversed across the original edges (when data sharing is enabled). For the 20 benchmarks used, 22,023 jmp edges have been added on average per benchmark. The number of steps saved by these jmp edges is much larger than that of the original ones, by a factor of $28.6 \mathrm{X}$ on average. This implies that a large number of redundant traversals $\left(\# S \times \frac{R_{S}}{R_{S}+1}\right.$ for a benchmark) have been eliminated. Thus, PARCFL ${ }_{D}^{16}$ exhibits substantial improvements over PARCFL naive, with the superlinear speedups observed in _202_jess, _213_javac, _222_mpegaudio, batik, fop and tomcat.

The optimisation described in Section IV-A for adding jmp edges selectively to reduce synchronisation overhead is also useful for improving the performance. Fig. 7 reveals the

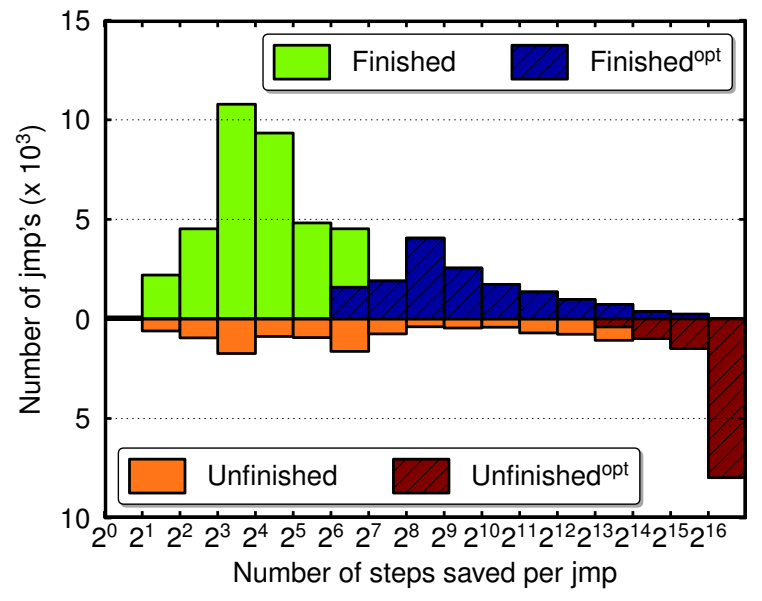

Fig. 7: Histograms of jmp edges (identified by the number of steps saved). Finished represents jmp edges in Fig. 3(a) and Unfinished jmp edges in Fig. 3(b). Finished ${ }^{\mathrm{opt}}$ (Unfinished ${ }^{\mathrm{opt}}$ ) is the version of Finished (Unfinished) with the selective optimisation described in Section IV-A being applied.

histograms of added jmp edges with and without this optimisation. In the absence of such optimisation, many jmp edges representing relatively short paths are also added, causing PARCFL $_{D Q}^{16}$ to drop from $16.2 \mathrm{X}$ to $12.4 \mathrm{X}$ on average.

3) Effectiveness of Query Scheduling: When query scheduling is also enabled, queries are grouped and reordered to increase early terminations made. PARCFL ${ }_{D Q}^{16}$ achieves superlinear speedups in two more benchmarks than $\operatorname{PARCFL}_{D}^{16}$ : avrora and sunflow. PARCFL ${ }_{D Q}^{16}$ is faster than $\operatorname{PARCFL}_{D}^{16}$ as the average speedup goes up from $13.4 \mathrm{X}$ to $16.2 \mathrm{X}$.

To understand its effectiveness, some statistics are given in Columns $11-13$ in Table I. For a benchmark, $\overline{S_{g}}$ gives the average number of queries in a group, \#ETs is the number of early terminations found without query scheduling, and $R_{E T}$ is the ratio of \#ETs obtained with query scheduling over \#ETs obtained without query scheduling. On average, our 


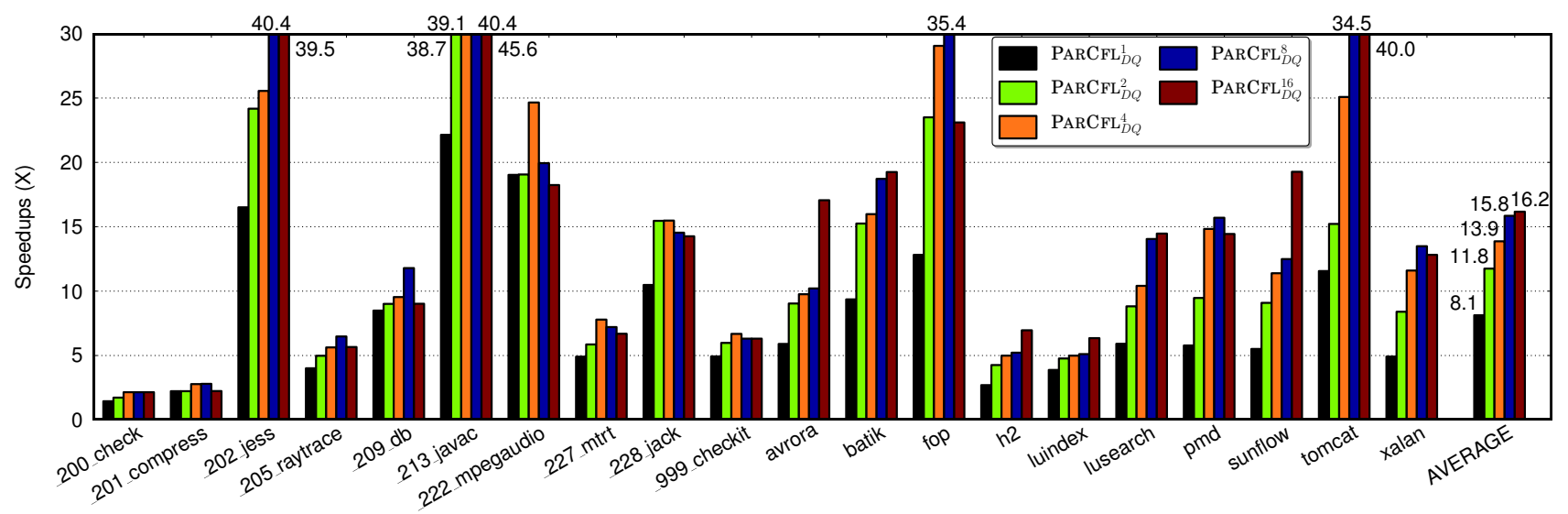

Fig. 8: Speedups of our parallel modes with different numbers of threads normalised with respect to SEQCFL.

query scheduling scheme leads to $35 \%$ more early terminations, resulting in more redundant traversals being eliminated.

4) Scalability: To see the scalability of our parallel implementation, Fig. 8 plots its speedups with a few thread counts over the baseline. PARCFL ${ }_{D Q}^{1}$ achieves an average speedup of $8.1 \mathrm{X}$, due to data sharing and query scheduling. Our parallel solution scales well to 8 threads for most benchmarks. When moving from 8 to 16 threads, PARCFL ${ }_{D Q}^{16}$ suffers some performance drops over $\mathrm{PARCFL}_{D Q}^{8}$ in some benchmarks (with _209_db being the worst case at $31 \%$ ). However PARCFL ${ }_{D Q}^{16}$ is still slightly faster than $\operatorname{PARCFL}_{D Q}^{8}$ on average.

5) Memory Usage: As garbage collection is enabled, it is difficult to monitor memory usage precisely. By avoiding redundant graph traversals, $\mathrm{PARCFL}_{D Q}^{16}$ reduces the memory usage by SEQCFL (the open-source sequential implementation [18]) by $35 \%(32 \%)$ in terms of the peak memory usage, despite the extra memory required for storing jmp edges. In the worst case attained at tomcat (fop), PARCFL ${ }_{D Q}^{16}$ consumes $103 \%(118 \%)$ of the memory consumed by SEQCFL ([18]).

\section{RELATED WORK}

\section{A. CFL-Reachability-Based Pointer Analysis}

In the sequential setting, there is no shortage of optimisations on improving the performance of demand-driven CFL-reachability-based pointer analysis. To ensure quick response, queries are commonly processed under budget constraints [17], [18], [19], [26], [27]. In addition, refinementbased schemes [18], [19] can be effective for certain clients, e.g., type casting if field-sensitivity is gradually introduced. Summary-based schemes avoid redundant graph traversals by reusing the method-local points-to relations summarised statically [26] or on-demand [17], achieving up to $3 X$ speedups. Must-not-alias information obtained during a pre-analysis can be exploited to yield an average speedup of $3 \mathrm{X}$ through reducing unnecessary alias-related computations [25]. Incremental techniques [6], [16], which are tailored for scenarios where code changes are small, take advantage of previously computed CFL-reachable paths to avoid unnecessary reanalysis.
Unlike these efforts on sequential CFL-reachability-based pointer analysis, this paper introduces the first parallel solution on multicore processors with significantly better speedups.

\section{B. Parallel Pointer Analysis}

In recent years, there have been a number of attempts on parallelising pointer analysis algorithms for analysing $\mathrm{C}$ or Java programs on multi-core CPUs and/or GPUs [3], [7], [8], [9], [10], [14], [20]. As compared in Table II, all these parallel solutions are different forms of Andersen's pointer analysis [2] with varying precision considerations in terms of context-, flow- and field-sensitivity. Our parallel solution is the only one that can be performed on-demand based on CFL-reachability.

Méndez-Lojo et al. [8] introduced the first parallel implementation of Andersen's pointer analysis for C programs. While being context- and flow-insensitive, their parallel analysis is field-sensitive, achieving a speedup of up to $3 \mathrm{X}$ on eight CPU cores. The same baseline sequential analysis was later parallelised on a Tesla C2070 GPU [7] (achieving an average speedup of $7 \mathrm{X}$ with 14 streaming multiprocessors) and a CPUGPU system [20] (boosting the CPU-only parallel solution by $51 \%$ and the GPU-only parallel solution by $79 \%$ on average).

Edvinsson et al. [3] described a parallel implementation of Andersen's analysis for Java programs, achieving a maximum speedup of $4.4 \mathrm{X}$ on eight CPU cores. Their analysis is fieldinsensitive but flow-sensitive (only partially since it does not perform strong updates). Recently, both field- and flowsensitivity (with strong updates for singleton objects) are handled for C programs on multi-core CPUs [9] and GPUs [10]. The speedups are up to $2.6 \mathrm{X}$ on eight $\mathrm{CPU}$ cores and $7.8 \mathrm{X}$ (with precision loss) on a Tesla C2070 GPU, respectively.

Putta and Nasre [14] use replications of points-to sets to enable more constraints (i.e., more pointer-manipulating statements) to be processed in parallel. Their context-sensitive implementation of Andersen-style analysis delivers an average speedup of $3.4 \mathrm{X}$ on eight CPU cores.

This paper presents the first parallel implementation of demand-driven CFL-reachability-based pointer analysis, achieving an average speedup of $16.2 \mathrm{X}$ for a set of 20 Java 


\begin{tabular}{|c|c|c|c|c|c|c|c|}
\hline \multirow{2}{*}{ Analysis } & \multirow{2}{*}{ Algorithm } & \multirow{2}{*}{ On-demand } & \multicolumn{3}{|c|}{ Sensitivity (Precision) } & \multirow{2}{*}{ Applications } & \multirow{2}{*}{ Platform } \\
\hline & & & Context & Field & Flow & & \\
\hline [8] & Andersen's [2] & $x$ & $x$ & $\checkmark$ & $x$ & $\mathrm{C}$ & $\mathrm{CPU}$ \\
\hline [3] & Andersen's [2] & $x$ & $x$ & $x$ & $J^{*}$ & Java & CPU \\
\hline [7] & Andersen's [2] & $x$ & $x$ & $\boldsymbol{V}$ & $x$ & $\mathrm{C}$ & GPU \\
\hline [14] & Andersen's [2] & $x$ & $\boldsymbol{V}$ & $x$ & $x$ & $\mathrm{C}$ & $\mathrm{CPU}$ \\
\hline [9] & Andersen's [2] & $x$ & $x$ & $\boldsymbol{\nu}$ & $\boldsymbol{V}$ & $\mathrm{C}$ & $\mathrm{CPU}$ \\
\hline$[10]$ & Andersen's [2] & $x$ & $x$ & $\boldsymbol{V}$ & $\boldsymbol{V}$ & $\mathrm{C}$ & GPU \\
\hline [20] & Andersen's [2] & $x$ & $x$ & $\boldsymbol{\nu}$ & $x$ & $\mathrm{C}$ & CPU-GPU \\
\hline this paper & CFL-Reachability [15] & $\checkmark$ & $\checkmark$ & $\checkmark$ & $x$ & Java & $\mathrm{CPU}$ \\
\hline
\end{tabular}

*: Partial flow-sensitivity without performing strong updates

TABLE II: Comparing different parallel pointer analysis.

programs on $16 \mathrm{CPU}$ cores. Based on a version of CFLreachability-based pointer analysis for $\mathrm{C}$ [27], our parallel solution is expected to generalise to $\mathrm{C}$ programs as well.

\section{Parallel Graph Algorithms}

There are many parallel graph algorithms, including breadth-first search (BFS) [1], [4], [11], minimum-cost path [12] and flow analysis [13]. However, parallelising CFLreachability-based pointer analysis poses different challenges. The presence of both context- and field-sensitivity that is enforced during graph traversals makes it hard to avoid redundant traversals efficiently, limiting the amount of parallelism exploited (especially within a single query). Exploiting interquery parallelism, this work has demonstrated significant performance benefits that can be achieved on parallelising CFLreachability-based pointer analysis on multi-core CPUs.

\section{CONCLUSION}

This paper presents the first parallel implementation of CFLreachability-based pointer analysis on multi-core CPUs. Despite the presence of redundant graph traversals, this demanddriven analysis is non-trivial to parallelise due to the dependences introduced by context- and field-sensitivity during graph traversals. We have succeeded in parallelising it by using (1) a data sharing scheme that enables the concurrent query-processing threads to avoid traversing earlier discovered paths via graph rewriting and (2) a query scheduling scheme that allows more redundancies to be eliminated based on the dependences statically estimated among the queries to be processed. For a set of 20 Java benchmarks evaluated, our parallel implementation significantly boosts the performance of a state-of-the-art sequential implementation with an average speedup of $16.2 \mathrm{X}$ on $16 \mathrm{CPU}$ cores.

\section{ACKNOWLEDGMENT}

This work is supported by Australian Research Grants, DP110104628 and DP130101970.

\section{REFERENCES}

[1] Virat Agarwal, Fabrizio Petrini, Davide Pasetto, and David A. Bader. Scalable graph exploration on multicore processors. In $S C$, pages 1-11, 2010.

[2] Lars Ole Andersen. Program analysis and specialization for the C programming language. PhD Thesis, University of Copenhagen, 1994.

[3] Marcus Edvinsson, Jonas Lundberg, and Welf Löwe. Parallel points-to analysis for multi-core machines. In HiPEAC, pages 45-54, 2011.
[4] Sungpack Hong, Tayo Oguntebi, and Kunle Olukotun. Efficient parallel graph exploration on multi-core CPU and GPU. In PACT, pages 78-88, 2011.

[5] Ondřej Lhoták and Laurie Hendren. Context-sensitive points-to analysis: Is it worth it? In $C C$, pages 47-64, 2006.

[6] Yi Lu, Lei Shang, Xinwei Xie, and Jingling Xue. An incremental pointsto analysis with CFL-reachability. In $C C$, pages 61-81, 2013.

[7] Mario Méndez-Lojo, Martin Burtscher, and Keshav Pingali. A GPU implementation of inclusion-based points-to analysis. In $P P O P P$, pages 107-116, 2012.

[8] Mario Méndez-Lojo, Augustine Mathew, and Keshav Pingali. Parallel inclusion-based points-to analysis. In OOPSLA, pages 428-443, 2010.

[9] Vaivaswatha Nagaraj and R. Govindarajan. Parallel flow-sensitive pointer analysis by graph-rewriting. In PACT, pages 19-28, 2013.

[10] Rupesh Nasre. Time- and space-efficient flow-sensitive points-to analysis. TACO, 10(4):39, 2013.

[11] Robert Niewiadomski, José Nelson Amaral, and Robert C. Holte. A parallel external-memory frontier breadth-first traversal algorithm for clusters of workstations. In ICPP, pages 531-538, 2006.

[12] Robert Niewiadomski, José Nelson Amaral, and Robert C. Holte. Sequential and parallel algorithms for frontier $\mathrm{A}^{*}$ with delayed duplicate detection. In $A A A I$, pages 1039-1044, 2006.

[13] Tarun Prabhu, Shreyas Ramalingam, Matthew Might, and Mary W. Hall. EigenCFA: Accelerating flow analysis with GPUs. In POPL, pages 511522, 2011.

[14] Sandeep Putta and Rupesh Nasre. Parallel replication-based points-to analysis. In $C C$, pages $61-80,2012$

[15] Thomas Reps. Program analysis via graph reachability. Information and Software Technology, 40(11):701-726, 1998.

[16] Lei Shang, Yi Lu, and Jingling Xue. Fast and precise points-to analysis with incremental CFL-reachability summarisation: Preliminary experience. In ASE, pages 270-273, 2012.

[17] Lei Shang, Xinwei Xie, and Jingling Xue. On-demand dynamic summary-based points-to analysis. In $C G O$, pages 264-274, 2012.

[18] Manu Sridharan and Rastislav Bodík. Refinement-based contextsensitive points-to analysis for Java. In PLDI, pages 387-400, 2006.

[19] Manu Sridharan, Denis Gopan, Lexin Shan, and Rastislav Bodík. Demand-driven points-to analysis for Java. In OOPSLA, pages 59-76, 2005.

[20] Yu Su, Ding Ye, and Jingling Xue. Accelerating inclusion-based pointer analysis on heterogeneous CPU-GPU systems. In HiPC, pages 149-158, 2013.

[21] Yulei Sui, Yue Li, and Jingling Xue. Query-directed adaptive heap cloning for optimizing compilers. In $C G O$, pages 1-11, 2013.

[22] Yulei Sui, Ding Ye, and Jingling Xue. Static memory leak detection using full-sparse value-flow analysis. In ISSTA, pages 254-264, 2012.

[23] Yulei Sui, Ding Ye, and Jingling Xue. Detecting memory leaks statically with full-sparse value-flow analysis. IEEE Transactions on Software Engineering, 40(2):107 - 122, 2014.

[24] Raja Vallée-Rai, Phong Co, Etienne Gagnon, Laurie J. Hendren, Patrick Lam, and Vijay Sundaresan. Soot - a Java bytecode optimization framework. In CASCON, page 13, 1999.

[25] Guoqing Xu, Atanas Rountev, and Manu Sridharan. Scaling CFLreachability-based points-to analysis using context-sensitive must-notalias analysis. In ECOOP, pages 98-122, 2009.

[26] Dacong Yan, Guoqing Xu, and Atanas Rountev. Demand-driven contextsensitive alias analysis for Java. In ISSTA, pages 155-165, 2011.

[27] Xin Zheng and Radu Rugina. Demand-driven alias analysis for C. In POPL, pages 197-208. ACM, 2008. 